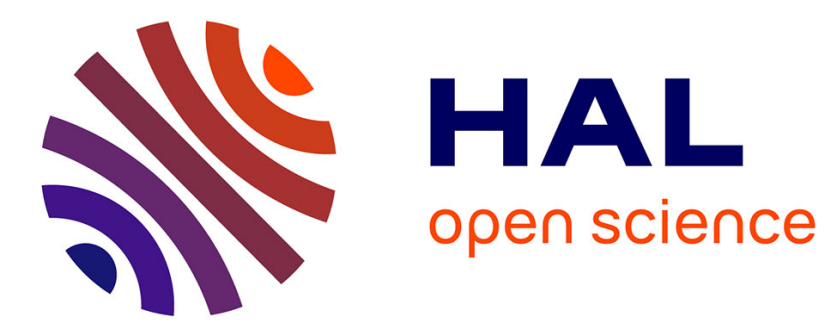

\title{
Développement des applications des supraconducteurs
}

H.A. Combet

\section{To cite this version:}

H.A. Combet. Développement des applications des supraconducteurs. Revue de Physique Appliquée, 1969, 4 (4), pp.557-557. 10.1051/rphysap:0196900404055700 . jpa-00243331

\section{HAL Id: jpa-00243331 https://hal.science/jpa-00243331}

Submitted on 1 Jan 1969

HAL is a multi-disciplinary open access archive for the deposit and dissemination of scientific research documents, whether they are published or not. The documents may come from teaching and research institutions in France or abroad, or from public or private research centers.
L'archive ouverte pluridisciplinaire HAL, est destinée au dépôt et à la diffusion de documents scientifiques de niveau recherche, publiés ou non, émanant des établissements d'enseignement et de recherche français ou étrangers, des laboratoires publics ou privés. 


\title{
DÉVELOPPEMENT DES APPLICATIONS DES SUPRACONDUGTEURS
}

\author{
H. A. COMBET,
}

C.N.E.T., Gentre de Recherches de Lannion, 22-Lannion.

En nous appuyant sur l'exemple américain [1], [2], [3], nous avons fait le point de l'évolution technologique qui s'est produite pendant l'année 1968 dans le domaine de la supraconductivité appliquée.

Les sujets abordés sont très variés, allant des petits dispositifs de laboratoire à l'électrotechnique lourde. On peut noter en particulier des progrès dans les directions suivantes.

I. Matériaux et aimants supraconducteurs. - Composé niobium-germanium-aluminium (température critique $20,7{ }^{\circ} \mathrm{K}$; champ critique de $200 \mathrm{kG}$ à $14 \mathrm{oK}$ ), couches minces de niobium-étain et de nitrures ternaires $\mathrm{Nb}-\mathrm{Ti}-\mathrm{N}$.

Stabilité thermique et électrique des fils supraconducteurs.

Continuation des travaux de développement de gros aimants : chambres à bulles des laboratoires d'Argonne et de Brookhaven, projet I.M.P. d'Oak Ridge, lévitation d'anneaux supraconducteurs, etc.

Applications spéciales : suspensions magnétiques pour véhicules, microscopes électroniques, etc.
II. Dispositifs pour fréquences élevées et instruments de mesure. - Fabrication de cavités hyperfréquences en niobium à coefficient de surtension très élevé et accélérateur supraconducteur.

Lignes de transmission coaxiales ou à ondes lentes, antennes de faible dimension.

Modulateurs supraconducteurs, magnétomètre à courant permanent.

Détecteurs rapides de radiation, présélecteurs accordables, etc.

III. Effet Josephson. - Amélioration de la fiabilité de jonctions évaporées (un gros effort a été fait dans ce domaine).

Électronique quantique à supraconducteurs, etc.

Toutes les applications des supraconducteurs (et en particulier les mémoires supraconductrices) n'ont pas été passées en revue, néanmoins on remarque de plus en plus un clivage certain entre le développement d'appareils de laboratoire à très hautes performances et la réalisation de grands projets industriels pour lesquels on se heurte constamment aux impératifs économiques.

\section{BIBLIOGRAPHIE}

[1] J. Appl. Phys., 1968, 39, 6.

[2] J. Appl. Phys., 1969, 40, 6.

[3] Newhouse (V. L.) et ATHeRTon (D. L.), Cryogenics, 1969, 9. 